\title{
Variables clínicas del parto en posición vertical y horizontal: revisión exhaustiva de literatura
}

\author{
Laura Cuy-Chaparro' (D), David Ricardo Salamanca² (D), Diana Margoth Riaño-Carreño ${ }^{3}$ (D), \\ Gloria Camargo-Villaba' ${ }^{1}$, Nohora Sánchez-Capacho' ${ }^{1}$ (D) Rosario Soto Bringas ${ }^{4}$
}

\begin{abstract}
RESUMEN para minimizar las complicaciones maternas y neonatales. describiendo los principales resultados en diferentes países del mundo. acuerdo con las características que presente cada paciente.

\footnotetext{
1 Universidad de Boyacá, Tunja, Colombia.

2 Universidad El Bosque, Grupo de Investigación GIRARE, IPS Rangel Rehabilitación, Bogotá, Colombia.

${ }^{3}$ Universidad de los Andes, Bogotá, Colombia.

${ }^{4}$ Universidad Andina del Cusco, Perú. Centro de Investigación en Salud Sexual y Reproductiva (Cinsare).
}

Introducción: El trabajo de parto representa el acontecimiento más importante durante la culminación del periodo de gestación y, por lo tanto, la adecuada elección de su posición, ya sea vertical u horizontal, resulta indispensable

Objetivo: Llevar a cabo una revisión de la literatura sobre el comportamiento clínico del parto vertical y horizontal,

Método: Revisión documental mediante la búsqueda de la literatura entre 2009 y 2020 en bases de datos especializadas. Resultados: La posición vertical se asoció con una menor duración del trabajo de parto, dolor referido y necesidad de episiotomía; no obstante, esta posición puede incrementar el riesgo de hemorragia posparto y daño perineal. Conclusión: La variedad de desenlaces obstétricos y neonatales asociados a las posiciones de parto vertical y horizontal hacen complejo definir una posición de parto óptima; por lo tanto, se recomienda la libre elección de

Palabras clave: parto obstétrico; trabajo de parto; complicaciones del trabajo de parto; presentación en trabajo de parto.

Autor de correspondencia: David Ricardo Salamanca, Correo electrónico: drsalamanca@uniboyaca.edu.co

Citar este artículo así:

Cuy-Chaparro L, Salamanca DR, Riaño-Carreño DM, Camargo-Villaba G, Sánchez-Capacho N, Soto Bringas R. Variables clínicas del parto en posición vertical y horizontal: revisión exhaustiva de literatura. Rev Investig Salud Univ Boyacá. 2021;8(1):91-111. https://doi.org/10.24267/23897325.601 


\title{
Clinical Behavior of Vertical and Horizontal Deliveries: Exhaustive Literature Review
}

\begin{abstract}
Introduction: Parturition represents the most important event during the culmination of the gestation period and, therefore, the appropriate choice of its position, whether vertical or horizontal, it is essential to minimize maternal and neonatal complications.
\end{abstract}

Objective: To carry out a review of the literature on the clinical behavior of vertical and horizontal delivery, describing the main results in different countries of the world.

Method: Document review by searching the literature between the years 2009 and 2020, available in Specialized Databases.

Results: The vertical position was associated with a shorter duration of labor obstetric, referred pain, and episiotomy; however, this position may increase the risk of postpartum hemorrhage and perineal damage.

Conclusion: The variety of obstetric and neonatal outcomes associated with vertical and horizontal delivery positions make it difficult to define an optimal delivery position and, therefore, free choice is recommended according to the characteristics of each patient.

Keywords: obstetric delivery; labor obstetric; obstetric labor complications; labor presentation. 


\section{Variáveis clínicas do parto em posição vertical e horizontal: revisão abrangente de literatura}

\section{RESUMO}

Introdução: O trabalho de parto representa o acontecimento mais importante no final do período gestacional e, portanto, a escolha apropriada da posição, seja vertical ou horizontal, é indispensável para minimizar as complicações maternas e neonatais.

Objetivo: Realizar uma revisão bibliográfica sobre o comportamento clínico do parto vertical e horizontal, descrevendo os principais resultados em diferentes países do mundo.

Método: Revisão documental através de pesquisa da literatura em bases de dados especializadas entre os anos 2009 e 2020.

Resultados: A posição vertical foi associada a uma duração mais curta do parto, dor referida e necessidade de episiotomía; contudo, esta posição pode aumentar o risco de hemorragia pós-parto e dano perineal.

Conclusão: A variedade de resultados obstétricos e neonatais associados às posições de parto vertical e horizontal torna complexa a escolha de uma posição de parto ótima; por conseguinte, recomenda-se a livre escolha de acordo com as caraterísticas de cada paciente.

Palavras-chave: parto obstétrico; trabalho de parto; complicações do trabalho de parto; apresentação fetal. 
Laura Cuy-Chaparro, David Ricardo Salamanca, Diana Margoth Riaño-Carreño, Gloria Camargo-Villaba, Nohora Sánchez-Capacho, Rosario Soto Bringas

\section{INTRODUCCIÓN}

Durante varios años, la posición seleccionada durante el trabajo de parto ha resultado de la interacción entre factores sociales, culturales y sanitarios. El desarrollo de políticas de salud encaminadas a la seguridad y el cuidado materno y neonatal, adoptadas especialmente por las sociedades occidentales, han convertido la posición horizontal, en cualquiera de sus variables: supina, litotomía o decúbito lateral, en una de las más utilizadas en la práctica ginecobstétrica actual (1), ya que resulta más conveniente para la inspección y vigilancia de la región perineal durante el trabajo de parto y el parto. Sin embargo, algunas pacientes informan que esta posición suele ser más dolorosa, incómoda y difícil de realizar (2).

La posición horizontal se ha empleado en las prácticas médicas desde cuando, en 1670, Hugh Chamberlen introdujo las pinzas obstétricas, y a partir de la implementación del uso de estribos, que, al combinar la inclinación lateral de la pelvis con una postura semirrecostada con la gestante sentada a unos 45 grados, resulta favorable para reducir la compresión aortocava (3). Entre otras ventajas reconocidas de esta posición, se resalta la facilidad en el acceso del cuidador al abdomen de la gestante para monitorear la frecuencia cardíaca fetal. Así mismo, se ha descrito que los médicos se sienten cómodos atendiendo el parto en posición horizontal, ya que han sido usualmente entrenados para realizar partos en esta, incluidos los partos vaginales espontáneos y asistidos, y es la posición de referencia convencional para las descripciones de los libros de texto sobre los mecanismos del parto vaginal (2).

No obstante, en diferentes regiones del mundo se ha adoptado la posición vertical en cualquiera de sus variables: sentada, cuclillas, de rodillas o de pie, atendiendo a los saberes ancestrales y las leyes de la física que respaldan su implementación (4). La posición vertical puede tener una influencia positiva sobre el trabajo de parto, debido al efecto de la gravedad que se genera sobre la perfusión uterina, que provoca contracciones uterinas más eficaces y una mejor alineación fetal en el canal del parto, que permite facilitar y agilizar el descenso y salida del neonato y la placenta (5).

Se ha observado que dicha posición ofrece beneficios obstétricos adicionales, particularmente en términos de reducción de tiempo del trabajo de parto y de intervención obstétrica. Entre las hipótesis que explicarían estos resultados, podemos vislumbrar que la posición vertical, así como la posición en cuclillas, está más cerca de la mejor posición teóricamente aceptada para el parto. Esta permite que el eje de progresión sea perpendicular al plano de entrada pélvica superior, al aplanar la bisagra dorsal (6). Para alcanzar estas condiciones óptimas, el plano de entrada pélvica debe estar perpendicular a la columna 
lumbar de acuerdo con la teoría obstétrica. La posición erguida también ayuda a que el útero se contraiga con más fuerza y eficacia y, como resultado, favorece que el feto adopte una mejor posición $(7,8)$. Las leyes de la gravedad y la acción mecánica aplicadas a la fisiología del parto influyen directamente en el feto, especialmente en su cabeza, y ello ayuda a su expulsión. Entre otras ventajas, se describe la reducción del sangrado obstétrico, los desgarros vaginales y un menor riesgo de cesárea, así como una rápida adaptación neonatal $(9,10)$.

Por lo anterior, el trabajo de parto en posición vertical representa un enfoque bien establecido y popular que data de la Antigüedad y que actualmente se práctica como método de elección en diferentes regiones; sin embargo, no existen hallazgos concluyentes que respalden un resultado superior de esta posición durante el parto en comparación con otras variantes $(11,12)$. La controversia sobre los efectos que la postura del trabajo de parto pueda tener en el desenlace materno y neonatal hacen complejo definir una posición de parto óptima (4).

Para que el personal sanitario mejore su atención a las mujeres en trabajo de parto, se requiere evidencia científica encaminada a proteger la salud materna, incluida la oportunidad de elegir cómo llevar a cabo sus procesos de trabajo de parto y parto. Por esto, el intento por identificar la posición más segura y recomendable durante el trabajo de parto y en el momento del parto ha suscitado la realización de diversos trabajos para demostrar los potenciales beneficios y riesgos descritos en las diferentes posiciones sobre la salud materna y fetal.

Dada la incertidumbre sobre la posición óptima para el parto, este artículo de revisión tuvo como objetivo evaluar los hallazgos disponibles sobre los potenciales beneficios y las desventajas de las diferentes posiciones durante el trabajo de parto y el parto en mujeres en diferentes países y continentes, mediante un análisis detallado de los datos epidemiológicos, obstétricos y neonatales descritos en los últimos diez años.

\section{METODOLOGÍA}

Se llevó a cabo una revisión exhaustiva de literatura científica para analizar las potenciales ventajas y desventajas del trabajo de parto según su posición, ya sea vertical u horizontal, existente desde 2009 hasta noviembre de 2020. La búsqueda se hizo a partir de las fuentes de información consultadas en las bases de datos PubMed Central, Web of Science, Embase, Redalyc y LILACS. Con base en los Medical Subject Headings (MeSH), se seleccionaron las palabras clave para la búsqueda en idiomas español e inglés, en combinación con el operador booleano AND, entre ellas: obstetric delivery; labor obstetric; obstetric labor complications; labor presentation. 
Se obtuvieron 94 publicaciones en total, de las cuales se seleccionaron 50 artículos publicados entre 2009 y 2020, disponibles en texto completo en idioma inglés y español que mostraban de forma detallada la información metodológica y los desenlaces maternos y neonatales expresados en duración del trabajo de parto, intensidad del dolor de acuerdo con la Escala de Calificación Numérica (NRS, por sus siglas en inglés), necesidad de parto instrumentalizado, presencia de hemorragia y desgarro perineal materno, ingreso de neonatos a la unidad de cuidados intensivos y tasa de mortalidad perinatal, teniendo en cuenta la posición durante el trabajo de parto. Todos los datos se almacenaron en el gestor bibliográfico Zotero. Se excluyeron 42 artículos que no evidenciaban descriptivamente los resultados relacionados con las ventajas y desventajas de las diferentes posiciones durante el trabajo de parto y parto.

\section{RESULTADOS}

Con base en la búsqueda, se presenta a continuación un análisis detallado de la epidemiología y el comportamiento clínico del parto vertical, comparado con el parto horizontal en diferentes países, estudiados por continente (véase el anexo).

\section{Continente europeo}

Un ensayo controlado y aleatorizado con 102 mujeres, realizado en Turquía entre diciembre de
2013 y mayo de 2014, comparó la posición en cuclillas usando barras frente a la posición supina en mujeres nulíparas. Encontró una reducción de $34 \mathrm{~min}$ en la duración de la segunda etapa del parto y una asociación con índices más bajos de dolor en las mujeres con parto en posición vertical respecto al grupo en posición supina, sin evidenciar diferencias significativas en la presentación de complicaciones maternas y fetales en ambos grupos (13).

Un estudio aleatorizado suizo con 1002 mujeres asignadas a dos grupos de estudio - parto en un asiento o parto vaginal en cualquier otra posición excepto en el asiento de parto- no evidenció una diferencia en la cantidad de partos vaginales instrumentalizados, laceraciones y edema perineal entre los dos grupos, pero sí se observó un aumento en las pérdidas sanguíneas entre $500 \mathrm{ml}$ y $1000 \mathrm{ml}$ en el grupo de parto en un asiento (14).

En 2013, un estudio italiano de cohorte observacional con mujeres primíparas comparó los efectos de las posiciones recostadas y verticales durante el trabajo de parto y encontró una importante disminución en el tiempo de duración de la segunda etapa de parto en el grupo de posiciones verticales, de aproximadamente $50 \mathrm{~min}$, respecto al grupo en posición decúbito supino. Otros resultados de este estudio mostraron que la posición vertical redujo la necesidad de un parto instrumentalizado y que hubo tasas de dolor más bajas (15). 
La revisión Cochrane publicada en 2017, que incluye datos de 30 ensayos controlados aleatorios o cuasialeatorios con la participación de 9015 mujeres primigrávidas y multigrávidas con más de 34 semanas de gestación asignadas a los grupos de posición de parto vertical (sentada, arrodillada o cuclillas) o posición de parto supina, sugirió como posibles beneficios de la postura vertical una disminución de 6,16 min promedio en la duración de la segunda etapa del trabajo de parto, principalmente en el grupo de primigrávidas, así como una reducción tanto en el dolor referido como en la tasa de episiotomías requerida por este grupo de gestantes. No se observaron diferencias entre los dos grupos en cuanto a la realización de cesáreas ni a la necesidad de transfusiones sanguíneas. Otros hallazgos evidenciaron que, aun cuando menos mujeres que adoptaron una posición de parto vertical necesitaron partos asistidos, existe un mayor riesgo de pérdida de sangre superior a $500 \mathrm{ml}$ y puede haber un mayor riesgo de desgarros perineales de segundo grado (2).

Las posiciones supina y vertical con anestesia epidural o sin esta no mostraron un efecto particular, en cuanto a la presentación de distocias de hombros, el ingreso de neonatos a la unidad de cuidados intensivos y la tasa de mortalidad perinatal. Sin embargo, los patrones anormales en la frecuencia cardiaca fetal fueron menores en el grupo de parto vertical $(R R=0,46)(2,16)$. La interpretación de los anteriores resultados debe ser cautelosa, teniendo en cuenta la diversidad metodológica y de sesgos presentada en cada uno de los ensayos incluidos en este estudio (2).

La evaluación de la posición materna adoptada durante el trabajo de parto y su efecto sobre la aparición de incontinencia urinaria en un estudio italiano con 656 mujeres primigrávidas que eligieron la posición cuclillas (grupo 1) y supina (grupo 2) para dar a luz evidenció una tasa de disfunción urinaria del $40,5 \%$ en el grupo 1 , comparada con el $48,9 \%$ en el grupo 2; adicionalmente, a través de un análisis multivariado se asoció la posición supina como único factor de riesgo para la aparición de síntomas de incontinencia urinaria en el posparto de estas gestantes (17).

Por otra parte, el riesgo de lesión anal asociado con las posiciones de parto sentada, litotomía, lateral, de pie sobre las rodillas, asiento de parto, supino, en cuclillas y de pie, evaluado en una cohorte de 113.279 partos únicos no instrumentalizados en Suecia, mostró que la posición de litotomía presenta un mayor riesgo de lesión anal, especialmente en las mujeres que han tenido hijos ( $R R=1,66 \%)$; mientras que la posición de pie representó el menor riesgo, independientemente de la paridad ( $R R=0,40 \%$ ) (18). La relación entre la posición materna durante el parto espontáneo y la presencia de desgarros del esfínter anal, según un estudio suizo realizado en 2012 , reveló 
que las posiciones en cuclillas y arrodilladas tienen tasas significativamente más altas $(2,9 \%$ y $2,1 \%$, respectivamente) de desgarros anales, en comparación con la posición al nacer en la cama $(1,0 \%)$ o en el agua $(0,9 \%)(19)$.

Un ensayo clínico llevado a cabo en los Países Bajos con 1646 mujeres que tuvieron un parto vaginal espontáneo comparó los resultados perineales entre mujeres en posición reclinada, semisentada y sentada, y no encontró diferencias significativas en cuanto a la presentación de perineo intacto entre los diferentes grupos de posiciones con $28,9 \%, 26,6 \%$ y $31,9 \%$, respectivamente. Por lo tanto, aunque se han descrito las lesiones obstétricas perineales como complicaciones maternas, la asociación entre las posiciones de parto y el daño perineal no es concluyente (20).

\section{Continente asiático}

La posición materna seleccionada para la segunda etapa del trabajo de parto es fundamental para facilitar el progreso del parto vaginal y su finalización de manera segura $(1,21)$. Teniendo en cuenta los variados resultados obtenidos en estudios clínicos llevados a cabo en el continente asiático, a la fecha, no existe información concluyente acerca de una posición de parto óptima de uso generalizado.
Un estudio observacional comparativo en Navi Mumbai (India) mostró una disminución promedio de 9 min en la duración de la segunda etapa del trabajo de parto tanto en primíparas como en multíparas que adoptaron una posición en cuclillas, así como un requerimiento menor de dosis de oxitocina, en comparación con la posición supina, sin diferencias en cuanto a las tasas de partos instrumentalizados, episiotomías, desgarros perineales y complicaciones fetales (22). Contrario a estos resultados, un ensayo clínico aleatorizado para mujeres primíparas que fueron derivadas al Hospital Taleghani, en Irán, no halló diferencias significativas en la duración media de la segunda etapa del trabajo de parto entre las posiciones de litotomía $(49,02 \mathrm{~min})$, cuclillas $(50,97 \mathrm{~min})$ y rodillas $(52,71 \mathrm{~min})$; tampoco en las puntuaciones de Apgar de 1 y 5 min entre los 3 grupos (23).

La percepción de dolor durante el parto varía de una mujer a otra, e influyen tanto variables físicas como psicológicas. Por lo tanto, aquellas posiciones que faciliten una mejor posición del feto en el canal vaginal tendrían un efecto positivo sobre la intensidad del dolor del parto. Un estudio aleatorizado realizado en los hospitales de Isfahán y Jahrom, en Irán, para evaluar el efecto de la posición del parto sobre la intensidad del dolor en la segunda etapa de trabajo de parto, asignó a 96 gestantes a los grupos de posiciones de litotomía, sentadas y en cuclillas. La intensidad del 
dolor experimentado con las posiciones litotomía y cuclillas fue mucho menor que en la posición sentada durante la fase latente de la segunda etapa de trabajo de parto; mientras que en la fase activa la intensidad del dolor fue menor en el grupo de posición en cuclillas $(6,14)$, en comparación con los grupos de litotomía $(7,4)$ y sentada $(7,59)(9)$. Estos resultados son apoyados por lo informado en otro estudio de 2012 en el Centro de Maternidad de Coimbatore (India), donde la posición semisentada redujo de manera significativa el dolor en primigrávidas, en contraste con la posición supina durante el trabajo de parto (3,4 y 7,86, respectivamente) (24). La evaluación del dolor y la duración de la segunda etapa del trabajo de parto en posiciones de rodillas, sentadas y supina en mujeres nulíparas que se llevó a cabo en el hospital Omolbanin de Mashhad (Irán) evidenció una menor intensidad del dolor en posición de rodillas $(65,32)$, comparado con las posiciones sentada $(65,32)$ y supina $(90,20)$, así como una menor duración de la segunda etapa del trabajo de parto en posición de rodillas, en relación con las otras dos posiciones estudiadas (25).

Un estudio multicéntrico chino evaluó los beneficios de la posición de manos y rodillas (grupo experimental, $n=446$ ) sobre la posición supina (grupo control, $\mathrm{n}=440$ ) durante el parto. Este evidenció una tasa más baja de episiotomías en el grupo experimental frente al grupo control $(1,8 \%$ y $37 \%$, respectivamente), así como tasas signifi- cativamente más altas de perineo intacto en el grupo experimental, sin encontrar diferencias significativas en la tasa de asfixia neonatal y las puntuaciones Apgar entre ambos grupos (10). Los anteriores resultados concuerdan con las conclusiones emitidas por un estudio previo que incluyó 113 mujeres chinas para investigar la posición de manos y rodillas (26).

\section{Continente americano}

En un estudio cualitativo llevado a cabo en Brasil que incluyó las percepciones de mujeres respecto a sus vivencias en el parto vaginal se determinó que los aspectos positivos de la posición horizontal fueron la reducción en su tiempo de duración y una mayor sensación de seguridad y apoyo por parte del personal médico; mientras que los aspectos negativos se relacionaron con la incomodidad y la dificultad para ejercer la fuerza. Dentro de algunos aspectos positivos de la posición vertical y los aspectos negativos de la posición horizontal se destacaron con mayor intensidad y frecuencia, y están de acuerdo con la evidencia científica (27).

En contraposición al anterior estudio, un ensayo clínico aleatorizado con 164 gestantes realizado en México evidenció que, aunque la postura vertical acorta el segundo periodo del trabajo de parto, se convierte en un factor de riesgo de desgarros vaginales $(R R=1,4) y$, por lo tanto, no 
Laura Cuy-Chaparro, David Ricardo Salamanca, Diana Margoth Riaño-Carreño, Gloria Camargo-Villaba,

Nohora Sánchez-Capacho, Rosario Soto Bringas

resulta claro su nivel de superioridad en cuanto al desarrollo de efectos adversos (28).

Por otra parte, en un estudio hecho en Perú con 85 gestantes no se observaron diferencias significativas en la aparición de complicaciones entre los dos tipos parto enunciados (29). Así mismo, otro ensayo clínico en Perú que evaluó distintas variables de los desenlaces obstétricos del parto vertical, comparándolos con el parto horizontal, determinó que variables como el volumen y la duración de sangrado fueron similares en ambos grupos. No obstante, otros aspectos, como el tiempo de expulsión, el requerimiento de episiotomía y el parto instrumentado fue menor en el parto vertical, por lo que en general se consideró que esta posición puede llegar a ser menos dolorosa, más cómoda y satisfactoria durante el trabajo de parto (30).

Entre las ventajas del parto vertical caracterizado en Perú se ha descrito también la reducción en el tiempo transcurrido de la fase activa al periodo expulsivo y una significante reducción en el número de episiotomías, aunque en otros estudios se han corroborado los resultados mencionados, donde el volumen de pérdida sanguínea en el parto vertical y horizontal no presentan diferencias estadísticamente significativas $(p=0,879)(31)$.

\section{Continente africano}

En la mayoría de las unidades de maternidad de África a las mujeres, a menudo, se les prohíbe estar de pie durante el trabajo de parto y el parto. En un estudio realizado en Benín, en el cual se estudió el efecto de una posición vertical (de pie, sentada, agachada) en comparación con la posición supina tradicional en el curso del parto y en los resultados perinatales y maternos (incluido el grado de satisfacción de las madres), se logró determinar que la posición vertical libre parece ser una opción eficaz y segura para el curso del trabajo de parto y el parto, y es más satisfactoria para las madres, pues se evidenció una duración más corta durante la segunda fase de labor, una menor necesidad de instrumentalización del parto y uso de episiotomía, así como una disminución en la anomalías de la frecuencia cardíaca fetal y la aparición de meconio en una proporción de casi tres veces $(2,9 \%$ frente a $8,9 \% ; p<0,01$ y $0,4 \%$ frente a $1,4 \% ; p<0,01$ ) en el grupo que adoptó esta posición (32). Sin embargo, el desenlace de lesión perineal y sangrado fue igual en ambos grupos

En un ensayo clínico aleatorizado en Sudáfrica no hubo un aumento en la hemorragia posparto, desgarros de tercer grado o hematoma vulvar en el grupo de posición vertical. La pérdida de volumen sanguíneo y la duración de la segunda etapa en los dos grupos fueron sorprendentemente similares, 
sin diferencias en la necesidad de intervención obstétrica. Las mujeres que tuvieron un parto vertical fueron sometidas a menos episiotomías y experimentaron menos dolor que las mujeres que dieron a luz en posición de litotomía, con un trauma perineal significativamente menor, por lo que estudios más recientes en ese país han sugerido que las mujeres en trabajo de parto deben poder seleccionar la posición de su elección, preferiblemente posiciones alternativas (incluidas posiciones verticales, de rodillas, en cuclillas y laterales), que eviten la litotomía. La elección de las posiciones de parto está respaldada por las pautas de atención de maternidad; sin embargo, las parteras continúan colocando de manera rutinaria a las mujeres en la posición de litotomía durante los partos de vértice normales, lo que provoca varios resultados maternos adversos (a saber, trabajo de parto prolongado, hemorragia posparto) y resultados neonatales adversos (como asfixia fetal y compromiso respiratorio) (33). En cuanto a la percepción del dolor, un estudio cualitativo, descriptivo y exploratorio en Ghana concluyó que la posición de cuclillas es una medida no farmacológica que mejora la percepción del dolor en la gestante (34).

La preferencia de la posición en litotomía se da en todo el continente. En Tanzania, un estudio cualitativo documentó que las mujeres utilizan la posición supina durante el parto, porque las enfermeras-parteras les indican que lo hagan, porque es la conocida y practicada universalmente, y la prefieren porque les brinda flexibilidad para monitorear continuamente el progreso del trabajo de parto y ayudar al parto de la manera más eficiente (35). No obstante, esta conducta está cambiando.

\section{Continente oceánico}

En un ensayo multicéntrico cuyo objetivo era valorar las tasas de partos domiciliarios, se determinó que en Australia esta modalidad es inferior al $<1 \%$ del total de los nacimientos $y$, en su mayoría, se asocian con partos en posición vertical (36). En un estudio retrospectivo en el sur de Australia que incluyó 1140 partos en el hogar y 298.860 partos en hospitales no se encontraron diferencias estadísticamente significativas en la tasa de mortalidad perinatal (7,9 frente a 8,2 por cada 1000 nacimientos), pero sí determinó un riesgo 7 veces mayor de muerte intraparto cuando se opta por el parto domiciliario (IC95\%: 1,53-35,87), principalmente por hemorragia masiva (37). Tal resultado fue similar a otros estudios reportados en la última década (38-41). Es importante resaltar que en este continente son escasos los estudios que comparan los tipos de parto según su posición, por lo que sería interesante caracterizar de forma más profunda a esta población y sus desenlaces maternos y neonatales. 
Laura Cuy-Chaparro, David Ricardo Salamanca, Diana Margoth Riaño-Carreño, Gloria Camargo-Villaba,

Nohora Sánchez-Capacho, Rosario Soto Bringas

\section{DISCUSIÓN}

Los estudios analizados mostraron que la duración del tiempo de trabajo de parto en las mujeres en posición vertical se redujo en la segunda etapa entre 6 y $50 \mathrm{~min}$, comparada con la posición supina $(2,15,28)$; así mismo, refirieron menor dolor y necesidad de episiotomía e instrumentalización del parto. Este último punto es controvertido, por la variabilidad de los hallazgos, pues muchos de los estudios no son concluyentes (14). Estos hallazgos son congruentes en los continentes de África, América, Europa y Asia, donde solo dos estudios documentan diferencias no significativas en la duración del segundo periodo de trabajo de parto $(23,29,42)$.

Respecto a los desenlaces perinatales, no hubo diferencia en las distocias ni en la tasa de mortalidad perinatal en el continente europeo; tampoco en tasas de asfixia neonatal y Apgar en el continente asiático (43).

Frente a la hemorragia posparto, se ha encontrado asociación entre las posiciones de parto vertical (parto semisentada y sentada) y una mayor pérdida de sangre entre las mujeres con daño perineal; sin embargo, la incidencia de hemorragia posparto relacionada con posiciones verticales y horizontales durante el parto sigue siendo inconstante, y difiere positiva o negativamente de una posición a otra $(14,44)$.
Hasta el momento, la información aportada por los anteriores estudios no permite establecer ninguna ventaja o desventaja específica en la adopción de una posición de parto, en particular sobre la gestante o el recién nacido $(15,45,46)$. Existen otros parámetros, como la intensidad del dolor, la instrumentalización del parto, la necesidad de conversión a cesárea y el traumatismo del esfínter anal, relacionados con diferentes posiciones de trabajo de parto que aún no se han estudiado lo suficiente $(15,47,48)$. En la actualidad, las diferencias entre las posiciones de partos no han sido concluyentes, por lo que no se recomienda un parto sobre otro, y se opta por permitir la elección de una posición libre para llevar a cabo el proceso de parto y se ha restringido el uso de la posición de litotomía, a fin de buscar un cambio de paradigma en la elección de posición del parto $(33,49,50)$.

\section{CONCLUSIÓN}

En la presente revisión se mostró un panorama general del comportamiento de las posiciones del parto en los diferentes continentes, con grandes diferencias entre cada uno de los estudios analizados, dadas por la tipología metodológica y los hallazgos de los desenlaces maternos y fetales. Así, se evidenciaron ventajas y desventajas de distinto tipo en cada posición. La decisión final de la elección del trabajo de parto debe orientarse según las oportunidades de atención médica, 
las características fisiológicas y antropométricas de la paciente, así como las comorbilidades y el nivel de confianza y seguridad percibida según la posición seleccionada y que, de esta manera, facilite el proceso de recuperación con una menor tasa de complicaciones asociadas. Se recomienda la realización de nuevos estudios con un mayor número de participantes, idealmente de tipo ensayo clínico y de cohorte prospectivo, de modo que se puedan controlar las variables en estudio $y$, así, obtener información objetiva de los distintos tipos de parto analizados.

\section{LIMITACIONES}

Teniendo en cuenta la alta tasa de variabilidad en los resultados obtenidos, con la presente revisión no es posible estandarizar una recomendación sobre el tipo de parto más indicado, ya que hacen falta estudios exploratorios más concluyentes.

\section{REFERENCIAS}

1. Kopas ML. A Review of evidence-based practices for management of the second stage of labor. J Midwifery Womens Health. 2014;59(3):26476. https://doi.org/10.1111/jmwh.12199

2. Gupta JK, Sood A, Hofmeyr GJ, Vogel JP. Position in the second stage of labour for women without epidural anaesthesia. Cochrane Database Syst Rev. 2017;25(5). https://doi. org/10.1002/14651858.CD002006.pub4
3. Dundes $L$. The evolution of maternal birthing position. Am J Public Health. 1987;77(5):63641. https://doi.org/10.2105/ajph.77.5.636

4. Huang J, Zang Y, Ren L-H, Li F-J, Lu H. A review and comparison of common maternal positions during the second-stage of labor. Int J Nurs Sci. 2019;6(4):460-7. https://doi. org/10.1016/j.ijnss.2019.06.007

5. Walker C, Rodríguez T, Herranz A, Espinosa $J A$, Sánchez E, Espuña-Pons M. Alternative model of birth to reduce the risk of assisted vaginal delivery and perineal trauma. Int Urogynecology J. 2012;23(9):1249-56. https:// doi.org/10.1007/s00192-012-1675-5

6. Desseauve D, Fradet L, Lacouture P, Pierre F. Position for labor and birth: state of knowledge and biomechanical perspectives. Eur J Obstet Gynecol Reprod Biol. 2017 ene;208:46-54. https://doi.org/10.1016/j.ejogrb.2016.11.006

7. Roberts JE. The "push" for evidence: management of the second stage. J Midwifery Womens Health. 2002;47(1):2-15. https://doi. org/10.1016/S1526-9523(01)00233-1

8. Hanson L. Second-stage labor care: challenges in spontaneous bearing down. J Perinat Neonatal Nurs. 2009;23(1):31-9. https://doi. org/10.1097/JPN.0b013e318196526b 
9. Valiani M, Rezaie M, Shahshahan Z. Comparative study on the influence of three delivery positions on pain intensity during the second stage of labor. Iran J Nurs Midwifery Res. 2016;21(4):372. https://doi. org/10.4103/1735-9066.185578

10. Zhang H-Y, Shu R, Zhao N-N, Lu Y-J, Chen $\mathrm{M}$, Li $\mathrm{Y}-\mathrm{X}$, et al. Comparing maternal and neonatal outcomes between hands-and-knees delivery position and supine position. Int J Nurs Sci. 2016;3(2):178-84. doi.org/10.1016/j. ijnss.2016.05.001

11. Chandler J, Clarke M, McKenzie J, Boutron I, Welch V. Cochrane Methods. Cochrane Database Syst Rev. 2016(10 Supl 1). https:// doi.org/10.1002/14651858.CD201601

12. What's your position on giving birth? Drug Ther Bull. 2018;56(8):85. https://doi. org/10.1136/dtb.2018.8.0649

13. Moraloglu O, Kansu-Celik H, Tasci $Y$, Karakaya $B K$, Yilmaz $Y$, Cakir $E$, et al. The influence of different maternal pushing positions on birth outcomes at the second stage of labor in nulliparous women. J Matern-Fetal Neonatal Med Off J Eur Assoc Perinat Med Fed Asia Ocean Perinat Soc Int Soc Perinat Obstet. 2017;30(2):245-9. https://doi.org/10.3109/14 767058.2016.1169525
14. Pop VJM, Pommer AM, Pop-Purceleanu M, Wijnen HAA, Bergink V, Pouwer F. Development of the Tilburg Pregnancy Distress Scale: the TPDS. BMC Pregnancy Childbirth. 2011;11:80. https://doi.org/10.1186/1471-2393-11-80

15. Gizzo S, Di Gangi S, Noventa M, Bacile V, Zambon A, Nardelli GB. Women's choice of positions during labour: return to the past or a modern way to give birth? A cohort study in Italy. BioMed Res Int. 2014;2014:638093. https://doi.org/10.1155/2014/638093

16. Kemp E, Kingswood CJ, Kibuka M, Thornton JG. Position in the second stage of labour for women with epidural anaesthesia. Cochrane Database of Systematic Reviews. 2013. https:// doi.org/10.1002/14651858.CD008070.pub2

17. Serati $M$, Di Dedda MC, Bogani G, Sorice $P$, Cromi A, Uccella $S$, et al. Position in the second stage of labour and de novo onset of post-partum urinary incontinence. Int Urogynecology J. 2016;27(2):281-6. https:// doi.org/10.1007/s00192-015-2829-z

18. Elvander C, Ahlberg M, Thies-Lagergren L, Cnattingius S, Stephansson O. Birth position and obstetric anal sphincter injury: a populationbased study of 113000 spontaneous births. BMC Pregnancy Childbirth. 2015;15:252. https://doi.org/10.1186/s12884-015-0689-7 
19. Haslinger C, Burkhardt T, Stoiber B, Zimmermann R, Schäffer L. Position at birth as an important factor for the occurrence of anal sphincter tears: a retrospective cohort study. J Perinat Med. 2015;43(6):715-20. https://doi. org/10.1515/jpm-2014-0172

20. De Jonge A, Van Diem MT, Scheepers PLH, Buitendijk SE, Lagro-Janssen ALM. Risk of perineal damage is not a reason to discourage a sitting birthing position: a secondary analysis. Int J Clin Pract. 2010;64(5):611-8. https://doi. org/10.1111/j.1742-1241.2009.02316.x

21. ljang YP, Cumber SNN, Nkfusai CN, Venyuy MA, Bede F, Tebeu PM. Awareness and practice of birth preparedness and complication readiness among pregnant women in the Bamenda Health District, Cameroon. BMC Pregnancy Childbirth. 2019;19(1):371. https:// doi.org/10.1186/s12884-019-2511-4

22. Dani A, V R B, Sawant G, S CS. Comparative study of squatting position vs dorsal recumbent position during second stage of labour. J Evid Based Med Healthc. 2015;2(54):8769-73. https://doi.org/10.18410/jebmh/2015/1223

23. Amiri L, Shirazi V, Rajabalipoor F. The effects of different positioning on the duration of the second stage of labor in primiparous women. J Zanjan Univ Med Sci Health Serv.
2012;20(80):105-14. http://zums.ac.ir/journal/ article-1-1836-en.html

24. Santhi, Anuratha, Kokilavani. Effectiveness of semi sitting position during 2 nd stage of labour on maternal and neonatal outcomes among primigravida. Nurs J India. 2012;103(6):272-5. PMID: 23923599.

25. Azhari S, Khalilian H Muvahhed, Tara F, Esmaeli $\mathrm{H}$. Comparison the effect of sitting and kneeling positions during the second stage of labor on pain and duration of second stage of labor in nulliparous women. IJOGI. 2013;15(38). https://doi.org/10.22038/ ijogi.2013.519

26. Guo X, Zhang H. Hands and knees position delivery on maternal and neonatal outcomes. J Hainan Med Coll. 2011;811-3.

27. Gayeski ME, Brüggemann OM. Puerperal women's perceptions on vertical and horizontal deliveries. Rev Lat Am Enfermagem. 2009;17(2):153-9. https://doi.org/10.1590/ S0104-11692009000200003

28. Calvo AO, Flores RAL, Morales GVE. Comparación de resultados obstétricos y perinatales del parto en postura vertical versus supina. Ginecol Obstet Mex [internet]. 2013;81(01):1-10. Disponible en: https:// 
www.medigraphic.com/pdfs/ginobsmex/gom2013/gom131b.pdf

29. Castillo M, Cahuata A, Calle A. Complicaciones del parto vertical en el Instituto Nacional Materno Perinatal de Lima, Perú. Horiz Med. 2014;14(1):31-7.

30. Calderón C, Bravo J, Albinagorta R. Parto vertical: retornando a una costumbre ancestral. Rev Per Ginecol Obstet. 2010;54:49-57.

31. Panduro L. Parto vertical con adecuación intercultural en relación al parto horizontal en gestantes atendidas en el hospital amazónico período mayo-agosto 2012 [tesis de doctorado en internet]. San Isidro, Perú: Universidad Nacional Hermilio Valdizán; 2016. Disponible en: http://repositorio.unheval.edu.pe/handle/ UNHEVAL/1777

32. Dénakpo J, Lokossou A, Tonato-Bagnan JA, Alao J, Hounkpatin B, Komongui DG, et al. L'accouchement en position libre peut-il être une solution de rechange à l'accouchement en position classique dans les salles de naissance en Afrique : résultats d'une étude prospective à Cotonou au Bénin. J Obstet Gynaecol Can. 2012;34(10):947-53.

33. Musie MR, Peu MD, Bhana-Pema V. Factors hindering midwives' utilisation of alternative birth positions during labour in a selected public hospital. Afr J Prim Health Care Fam Med. 2019;11(1). https://doi.org/10.4102/ phcfm.v11i1.2071

34. Aziato L, Acheampong AK, Umoar KL. Labour pain experiences and perceptions: a qualitative study among post-partum women in Ghana. BMC Pregnancy Childbirth. 2017;17(1):73. https://doi.org/10.1186/s12884-017-1248-1

35. Mselle LT, Eustace L. Why do women assume a supine position when giving birth? The perceptions and experiences of postnatal mothers and nurse-midwives in Tanzania. BMC Pregnancy Childbirth. 2020;20(1):36. https:// doi.org/10.1186/s12884-020-2726-4

36. Roome S, Hartz D, Tracy S, Welsh A. Why such differing stances? A review of position statements on home birth from professional colleges. BJOG Int J Obstet Gynaecol. 2016;123(3):376-82. https://doi. org/10.1111/1471-0528.13634

37. Kennare RM, Keirse MJNC, Tucker GR, Chan AC. Planned home and hospital births in South Australia, 1991-2006: differences in outcomes. Med J Aust. 2010;192(2):76-80.

38. Davies-Tuck ML, Wallace EM, Davey M-A, Veitch V, Oats J. Planned private homebirth 
in Victoria 2000-2015: a retrospective cohort study of Victorian perinatal data. BMC Pregnancy Childbirth. 2018;18(1):357. https:// doi.org/10.1186/s12884-018-1996-6

39. Naylor Smith J, Taylor B, Shaw K, Hewison A, Kenyon S. I didn't think you were allowed that, they didn't mention that. A qualitative study exploring women's perceptions of home birth. BMC Pregnancy Childbirth. 2018;18(1):105. https://doi.org/10.1186/s12884-018-1733-1

40. Keedle H, Schmied V, Burns E, Dahlen HG. Women's reasons for, and experiences of, choosing a homebirth following a caesarean section. BMC Pregnancy Childbirth. 2015;15:206. https://doi.org/10.1186/s12884-015-0639-4

41. Homer CSE, Cheah SL, Rossiter C, Dahlen HG, Ellwood D, Foureur MJ, et al. Maternal and perinatal outcomes by planned place of birth in Australia 2000 - 2012: a linked population data study. BMJ Open. 2019;9(10):e029192. https://doi.org/10.1136/bmjopen-2019029192

42. Martin de Vega RA, Feijoo Iglesias MB, Magdaleno del Rey G, Rodriguez Ferrer RM, Ruiz Rey AM. Effect of Sims modified posture on the rotation and descent of the fetus in women during their first delivery with epidural analgesia. NURE. 2011;8(55):1-11.
43. Vargas Londoño VF, Rodríguez Castiblanco JP, Corredor Acosta MT, Vallejo Astudillo NM. Ventajas y desventajas del parto vertical en contraste con el parto horizontal: una revisión de la literatura. Investig Enferm Imagen Desarro. 2018;20(1). https://doi.org/10.11144/ Javeriana.ie20-1.vdpv

44. Oyelese $Y$, Ananth CV. Postpartum hemorrhage: epidemiology, risk factors, and causes. Clin Obstet Gynecol. 2010;53(1):147-56. https:// doi.org/10.1097/GRF.0b013e3181cc406d

45. Simarro M, Espinosa JA, Salinas C, Ojea R, Salvadores $\mathrm{P}$, Walker $\mathrm{C}$, et al. A Prospective randomized trial of postural changes vs passive supine lying during the second stage of labor under epidural analgesia. Med Sci Basel Switz. 2017;5(1). https://doi.org/10.3390/medsci5010005

46. Zaibunnisa, Ara F, Ara B, Kaker P, Aslam M. Child birth: comparison of complications between lithotomy position and squatting position during. Professional Med J. 2015;22(4):390-4.

47. Nilsen $E$, Sabatino $H$, Lopes MHB de M. [The pain and behavior of women during labor and the different positions for childbirth]. Rev Esc Enferm U P. 2011 jun;45(3):557-65. https://doi. org/10.1590/s0080-62342011000300002 
48. Jones $\mathrm{L}$, Othman $\mathrm{M}$, Dowswell $\mathrm{T}$, Alfirevic Z, Gates $S$, Newburn M, et al. Pain management for women in labour: an overview of systematic reviews. Cochrane Database Syst Rev. 2012 mar 14;(3):CD009234. https://doi. org/10.1002/14651858.CD009234.pub2

49. Zileni BD, Glover $\mathrm{P}$, Jones $\mathrm{M}$, Teoh $\mathrm{K}-\mathrm{K}$, Zileni $\mathrm{CW}$, Muller A. Malawi women's knowledge and use of labour and birthing positions: a cross-sectional descriptive survey. Women Birth J Aust Coll Midwives. febrero de 2017;30(1):e1-8. https:// doi.org/10.1016/j.wombi.2016.06.003

50. World Health Organization. WHO recommendations: intrapartum care for a positive childbirth experience [internet]. Geneva; 2018. Disponible: http://apps.who.int/iris/bitstream/handle/10665 /260178/9789241550215-eng.pdf;jsessionid =86C8EFA4ECC29CC8548F72E0006B7904?sequence $=1$

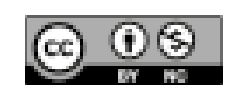

Esta obra está bajo una licencia de Creative Commons Reconocimiento-NoComercial 4.0 Internacional 
Anexo. Epidemiología y comportamiento clínico del parto vertical comparado con el parto horizontal en diferentes países, según cada continente

\begin{tabular}{|c|c|c|}
\hline Autores, año y país & $\begin{array}{l}\text { Tipo de estudio y tamaño } \\
\text { de muestra }\end{array}$ & Principales resultados \\
\hline & & Europa \\
\hline \multirow{3}{*}{$\begin{array}{l}\text { Moraloglu et al., } \\
\text { 2017. Turquía (13) }\end{array}$} & \multirow{3}{*}{$\begin{array}{l}\text { Ensayo controlado aleatorio } \\
\text { con } 102 \text { mujeres }\end{array}$} & Posición del trabajo de parto: cuclillas-supina \\
\hline & & Duración del trabajo de parto: $21,02 \pm 5,60 \mathrm{~min}-55,40 \pm 6,91 \mathrm{~min}$ \\
\hline & & Valoración de dolor: posición supina asociada a mayor nivel de dolor \\
\hline $\begin{array}{l}\text { Pop et al., } 2011 . \\
\text { Suecia (14) }\end{array}$ & $\begin{array}{l}\text { Ensayo controlado aleatorio } \\
\text { con } 419 \text { mujeres }\end{array}$ & $\begin{array}{l}\text { El parto en el asiento de parto no redujo el número de partos vaginales instrumen- } \\
\text { tales y se presentó un aumento en la pérdida de sangre entre } 500 \mathrm{ml} \text { y } 1000 \mathrm{ml} \text { con } \\
\text { este tipo de posición }\end{array}$ \\
\hline \multirow{4}{*}{$\begin{array}{l}\text { Gizzo et al., } 2014 . \\
\text { Italia (15) }\end{array}$} & \multirow{4}{*}{$\begin{array}{l}\text { Cohorte observacional con } \\
225 \text { mujeres }\end{array}$} & Posición del trabajo de parto: reclinada-vertical \\
\hline & & Valoración de dolor: puntuación NRS 7,1-3,7 \\
\hline & & Complicaciones: distosia, $13,05 \%-0,7 \%$ \\
\hline & & Episiotomía: $100 \%-32,7 \%$ \\
\hline $\begin{array}{l}\text { Gupta et al. Multi- } \\
\text { céntrico (2) }\end{array}$ & Revisión sistemática & $\begin{array}{l}\text { Se sugieren como posibles beneficios de la posición erguida una reducción muy } \\
\text { pequeña en la duración de la segunda etapa del trabajo de parto, reducción en las } \\
\text { tasas de episiotomía y partos asistidos en mujeres con parto sin anestesia epidural }\end{array}$ \\
\hline $\begin{array}{l}\text { Kemp et al., } 2013 . \\
\text { Multicéntrico (16) }\end{array}$ & Revisión sistemática & $\begin{array}{l}\text { No se identificaron diferencias estadísticamente significativas entre las posiciones } \\
\text { erguida y reclinada en los resultados del parto quirúrgico, la duración de la segunda } \\
\text { etapa del trabajo de parto ni en ningún otro resultado materno o fetal importante. } \\
\text { No hay datos suficientes para decir algo concluyente sobre el efecto de la posición } \\
\text { para la segunda etapa del trabajo de parto en mujeres con analgesia epidural }\end{array}$ \\
\hline \multirow{3}{*}{$\begin{array}{l}\text { Serati et al., } 2014 . \\
\text { Italia (17) }\end{array}$} & \multirow{3}{*}{ Prospectivo con 716 mujeres } & Posición del trabajo de parto: erguida-supina \\
\hline & & Complicaciones: IU de novo, 40,5\%-48,9\% \\
\hline & & Episiotomía $30,4 \%-40,5 \%$ \\
\hline \multirow{5}{*}{$\begin{array}{l}\text { Elvander et al., } 2014 \\
\text { Suecia (18) }\end{array}$} & \multirow{5}{*}{$\begin{array}{l}\text { Descriptivo con } 113.279 \\
\text { mujeres }\end{array}$} & $\begin{array}{l}\text { Posición del trabajo de parto: 1) sentado, 2) litotomía, 3) lateral, 4) de pie sobre las } \\
\text { rodillas, 5) asiento de parto, 6) supino, 7) en cuclillas, 8) de pie. }\end{array}$ \\
\hline & & Lesión obstétrica del esfínter anal nulíparas: 5,7\% \\
\hline & & Lesión obstétrica del esfínter anal multíparas: 1,3\% \\
\hline & & Lesión obstétrica del esfínter anal parto vaginal después de una cesárea: 10,6\% \\
\hline & & Lesión obstétrica del esfínter anal nulíparas: litotomía; RR: 1,17 \\
\hline
\end{tabular}


Laura Cuy-Chaparro, David Ricardo Salamanca, Diana Margoth Riaño-Carreño, Gloria Camargo-Villaba, Nohora Sánchez-Capacho, Rosario Soto Bringas

\begin{tabular}{|c|c|c|}
\hline Autores, año y país & $\begin{array}{l}\text { Tipo de estudio y tamaño } \\
\text { de muestra }\end{array}$ & Principales resultados \\
\hline & & Europa \\
\hline \multirow{2}{*}{$\begin{array}{l}\text { Haslinger et al., } 2015 . \\
\text { Suiza (19) }\end{array}$} & \multirow{2}{*}{$\begin{array}{l}\text { Cohorte retrospectivo con } \\
7832 \text { mujeres }\end{array}$} & $\begin{array}{l}\text { Posición del trabajo de parto: 1) cama en el agua, 2) rodillas, 3) cuclillas en un } \\
\text { taburete bajo }\end{array}$ \\
\hline & & $\begin{array}{l}\text { Desgarros del esfínter anal: cuclillas ( } 2,9 \%) \text {, arodilladas }(2,1 \%) \text {, nacer en la cama } \\
(1,0 \%) \text {, nacer en el agua }(0,9 \%)\end{array}$ \\
\hline \multirow{3}{*}{$\begin{array}{l}\text { De Jonge et al., } 2010 . \\
\text { Países Bajos (20) }\end{array}$} & \multirow{3}{*}{$\begin{array}{l}\text { Análisis secundario con } 1646 \\
\text { mujeres }\end{array}$} & Posición del trabajo de parto: semisentada-sentada \\
\hline & & Episiotomía: semisentada (24,6\%); sentada $(11,8 \%)$ \\
\hline & & Daño del esfínter anal: semisentada (1,5\%); sentada $(3,4 \%)$ \\
\hline \multicolumn{3}{|r|}{ Asia } \\
\hline $\begin{array}{l}\text { Ijang et al., } 2015 . \\
\text { India }(21)\end{array}$ & $\begin{array}{l}\text { Comparativo observacional } \\
\text { con } 345 \text { mujeres }\end{array}$ & $\begin{array}{l}\text { La posición en cuclillas para la segunda etapa del trabajo de parto puede ser benefi- } \\
\text { ciosa para las mujeres, en términos de duración, necesidad y cantidad de oxitocina } \\
\text { requerida. No se encuentran diferencias significativas en la incidencia de complica- } \\
\text { ciones maternas y fetales }\end{array}$ \\
\hline $\begin{array}{l}\text { Dani et al., } 2010 . \\
\text { Irán (22) }\end{array}$ & $\begin{array}{l}\text { Ensayo clínico aleatorizado } \\
\text { con } 200 \text { mujeres }\end{array}$ & $\begin{array}{l}\text { No se encontraron diferencias significativas en la duración de la segunda etapa del } \\
\text { trabajo de parto entre los } 3 \text { grupos: litotomía, en cuclillas y de rodillas }\end{array}$ \\
\hline \multirow{4}{*}{$\begin{array}{l}\text { Valiani et al., } 2012 . \\
\text { Irán (9) }\end{array}$} & \multirow{4}{*}{$\begin{array}{l}\text { Ensayo clínico aleatorizado } \\
\text { con } 96 \text { mujeres }\end{array}$} & En la fase latente de la segunda etapa del trabajo de parto: \\
\hline & & Valoración del dolor: litotomía: 2,27; cuclillas: 2,48, y sentada: 5,33 \\
\hline & & En la fase activa de la segunda etapa del trabajo de parto: \\
\hline & & Valoración del dolor: litotomía: 7,41; cuclillas: 6,14; sentada: 7,59 \\
\hline \multirow{3}{*}{$\begin{array}{l}\text { Amiri et al., } 2012 . \\
\text { Irán }(23)\end{array}$} & \multirow{3}{*}{$\begin{array}{l}\text { Ensayo clínico con } 148 \\
\text { mujeres }\end{array}$} & Posición del trabajo de parto: litotomía-cuclillas-rodillas \\
\hline & & $\begin{array}{l}\text { Duración del trabajo de parto: litotomía: } 49,02 \text { min; cuclillas: 50,97 min; rodillas: } \\
52,71 \text { min }\end{array}$ \\
\hline & & Tasas de cesárea: litotomía: 5,76; cuclillas: 7,54; rodillas: 9,1 \\
\hline \multirow{3}{*}{$\begin{array}{l}\text { Zhang et al., } 2012 . \\
\text { China (10) }\end{array}$} & \multirow{3}{*}{$\begin{array}{l}\text { Ensayo clínico con } 446 \\
\text { mujeres }\end{array}$} & Posición del trabajo de parto: rodillas-sentada \\
\hline & & Episiotomía: rodillas: $1,8 \%$; sentada: $37,7 \%$ \\
\hline & & Desgarro perineal: rodillas: $56,3 \%$; sentada: $41,8 \%$ \\
\hline \multicolumn{3}{|r|}{ América } \\
\hline $\begin{array}{l}\text { Gayeski et al., } 2009 . \\
\text { Brasil (27) }\end{array}$ & $\begin{array}{l}\text { Estudio descriptivo con } 20 \\
\text { mujeres }\end{array}$ & $\begin{array}{l}\text { El parto en posición vertical resulta ser más cómodo; favorece la movilidad y redu- } \\
\text { ce el esfuerzo de expulsión, mientras que el parto horizontal genera mayor tasa de } \\
\text { seguridad }\end{array}$ \\
\hline $\begin{array}{l}\text { Calvo et al., } 2013 . \\
\text { México (28) }\end{array}$ & $\begin{array}{l}\text { Ensayo clínico aleatorizado } \\
\text { con } 164 \text { mujeres }\end{array}$ & $\begin{array}{l}\text { Aunque el parto vertical podría acortar el segundo periodo del trabajo de parto, se } \\
\text { considera que la mejor postura se asocia con aquella posición con la que la paciente } \\
\text { se siente más cómoda }\end{array}$ \\
\hline
\end{tabular}




\begin{tabular}{|c|c|c|}
\hline Autores, año y país & $\begin{array}{l}\text { Tipo de estudio y tamaño } \\
\text { de muestra }\end{array}$ & Principales resultados \\
\hline \multicolumn{3}{|r|}{ América } \\
\hline $\begin{array}{l}\text { Castillo et al., } 2014 . \\
\text { Perú (29) }\end{array}$ & $\begin{array}{l}\text { Ensayo clínico aleatorizado } \\
\text { con85 mujeres }\end{array}$ & $\begin{array}{l}\text { No se determinaron diferencias significativamente estadísticas entre el parto verti- } \\
\text { cal y el horizontal }\end{array}$ \\
\hline $\begin{array}{l}\text { Calderón et al., } 2010 \\
\text { Perú (30) }\end{array}$ & $\begin{array}{l}\text { Ensayo clínico con } 160 \\
\text { mujeres }\end{array}$ & $\begin{array}{l}\text { El parto vertical ofrece ventajas sobre el parto horizontal, ya que desencadena un } \\
\text { periodo expulsivo más corto, cómodo y satisfactorio y menos doloroso }\end{array}$ \\
\hline $\begin{array}{l}\text { Panduro et al., } 2016 . \\
\text { Perú (31) }\end{array}$ & $\begin{array}{l}\text { Estudio descriptivo con } 84 \\
\text { mujeres }\end{array}$ & $\begin{array}{l}\text { El volumen de sangrado entre el parto vertical y horizontal no presenta diferencias } \\
\text { estadísticamente significativas }\end{array}$ \\
\hline \multicolumn{3}{|r|}{ África } \\
\hline $\begin{array}{l}\text { Dénakpo et al., } 2012 . \\
\text { Benín (32) }\end{array}$ & $\begin{array}{l}\text { Comparativo observacional } \\
\text { con } 490 \text { mujeres }\end{array}$ & $\begin{array}{l}\text { La elección de una posición vertical libre parece ser una opción eficiente y segura } \\
\text { durante el trabajo de parto y el parto y es más satisfactorio para las madres. Los } \\
\text { desgarros perineales y cervicales son raros y ocurren en cantidades similares en } \\
\text { ambos grupos, y lo mismo puede decirse de los casos de hemorragia posparto }\end{array}$ \\
\hline $\begin{array}{l}\text { Musie et al., } 2019 . \\
\text { Sudáfrica (33) }\end{array}$ & $\begin{array}{l}\text { Estudio descriptivo cualitati- } \\
\text { vo con } 20 \text { mujeres }\end{array}$ & $\begin{array}{l}\text { La falta de habilidades y capacitación durante el programa de pregrado y posgrado } \\
\text { en partería contribuye a que las parteras sean incompetentes para utilizar posicio- } \\
\text { nes alternativas para el parto durante la práctica clínica }\end{array}$ \\
\hline $\begin{array}{l}\text { Aziato et al., } 2017 . \\
\text { Ghana (34) }\end{array}$ & $\begin{array}{l}\text { Estudio descriptivo cualitati- } \\
\text { vo con } 14 \text { mujeres }\end{array}$ & $\begin{array}{l}\text { Se concluye que es necesario que todos los profesionales de la salud manejen el } \\
\text { dolor del parto de manera efectiva teniendo en cuenta el contexto sociocultural }\end{array}$ \\
\hline $\begin{array}{l}\text { Mselle et al., } 2020 . \\
\text { Tanzania (35) }\end{array}$ & $\begin{array}{l}\text { Estudio descriptivo cualitati- } \\
\text { vo con } 23 \text { mujeres }\end{array}$ & $\begin{array}{l}\text { Las parteras comúnmente deciden las posiciones de parto para las mujeres en par- } \\
\text { to. La posición supina es la más conocida y aceptada por las gestantes }\end{array}$ \\
\hline \multicolumn{3}{|r|}{ Oceanía } \\
\hline $\begin{array}{l}\text { Kennare et al., } 2010 \\
\text { Australia (37) }\end{array}$ & $\begin{array}{l}\text { Estudio descriptivo retros- } \\
\text { pectivo con } 26 \text { mujeres }\end{array}$ & $\begin{array}{l}\text { Los partos domiciliarios presentan una tasa de mortalidad perinatal similar a la de } \\
\text { los partos hospitalarios planificados, aunque se asocian a un mayor riesgo de muer- } \\
\text { te intraparto }\end{array}$ \\
\hline
\end{tabular}

